\title{
Publisher's Note: Control of the electromagnetic environment of a quantum emitter by shaping the vacuum field in a coupled-cavity system [Phys. Rev. A 91, 063807 (2015)]
}

\author{
Robert Johne, Ron Schutjens, Sartoon Fattah poor, Chao-Yuan Jin, and Andrea Fiore
}

(Received 28 July 2016; published 5 August 2016)

DOI: 10.1103/PhysRevA.94.029902

This paper was published online on 4 June 2015 with an error in a label of Fig. 1(c), in the caption of Fig. 1, and in the text below Eq. (3). The $y$-axis label of Fig. 1(c) should read as " $\left|\alpha_{t}^{(1)}\right|$." The third sentence of the caption to Fig. 1 should read as "(c) Calculated $\left|\alpha_{t}^{(1)}\right|$, which governs the modulation of the QE-cavity coupling constant $g$." The second line under Eq. (3) should read as "detuning and the target cavity fraction $\left|\alpha_{t}^{(1)}\right|$ of the mode...." The paper has been corrected as of 28 July 2016. The figure and text are incorrect in the printed version of the journal. 